\title{
Inflammatory Markers and Adipokines Alter Adipocyte- derived ASP Production Through Direct and Indirect Immune Interaction
}

Authors

Affiliations

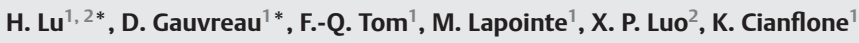

${ }^{1}$ Centre de Recherche Institut Universitaire de Cardiologie \& Pneumologie de Québec, Université Laval, Québec, Canada ${ }^{2}$ Department of Pediatrics, Tongji Hospital, HuaZhong University of Science and Technology, Wuhan, Hubei, P. R. China
Key words

- adipocytokines

- inflammation

obesity received $\quad 09.08 .2012$

first decision 01.11.2012

accepted 17.12.2012

Bibliography

DOI http://dx.doi.org/

10.1055/s-0032-1333231

Published online:

February 21, 2013

Exp Clin Endocrinol Diabetes

2013; 121: 194-200

(c) J. A. Barth Verlag in

Georg Thieme Verlag KG

Stuttgart · New York

ISSN 0947-7349

\section{Correspondence}

\section{H. Lu, MD, PhD}

Department of Pediatrics

Tongji Hospital

HuaZhong University of Science and Technology

Wuhan

Hubei

P. R. China

Tel.: + 86/27/8366 3315

Fax: + 86/27/8366 2393

huilinglu@yahoo.cn

\section{Abstract}

$\checkmark$

Obesity and related metabolic diseases are associated with chronic low-grade inflammation, characterized by increased pro-inflammatory proteins. Several studies have demonstrated increases in acylation stimulating protein (ASP) and its precursor protein C3 in obesity, diabetes and dyslipidemia. To evaluate the effects of acute inflammatory factors and adipokines on ASP production and potential mechanisms of action, 3T3-L1 adipocytes were treated for $24 \mathrm{~h}$ with adipokines, cytokines, macrophage-conditioned media and direct co-culture with J774 macrophages. ASP and C3 in the media were evaluated in relation to changes in adipocyte lipid metabolism (cellular triglyceride stores). Leptin, adiponectin, IL-10, LPS and TNF- $\alpha$ increased ASP production (151\%, 153\%, 190\%, 318\%, 134\%, $P<0.05$, respectively,). C5a and RANTES (Regulated and normal $\mathrm{T}$ cell expressed and secreted) decreased ASP production $(-34 \%,-47 \%, P<0.05)$, which was also associated with a decrease in the precursor protein C3 $(-39 \%$ to $-51 \%, P<0.01)$, while keratinocyte chemoattractant (KC; murine IL-8 ortholog) had no effect on ASP and C3 secretion. By contrast, apelin, omentin and visfatin also decreased ASP $(-27 \%,-49 \%,-22 \%, P<0.05)$, but without changes in precursor protein C3 secretion. Macrophage-conditioned media alone had little effect on C3 or ASP, while co-culture of adipocytes with macrophages markedly increased ASP and C3 production $(272 \%, 167 \%, P<0.05)$. These in vitro results suggest various metabolic hormones and inflammatory factors can affect ASP production through increased precursor C3 production and/or by changing the rate of $\mathrm{C} 3$ conversion to ASP. As an adipokine, ASP could constitute a new link between adipocytes and macrophages.

\footnotetext{
${ }^{*}$ Shared first author.
}

\section{Introduction}

$\nabla$

Obesity is now well recognized as being associated with a state of systemic low-grade inflammation [1,2]. During a situation of energy imbalance (increased caloric intake or diminished expenditure), adipocytes serve as energy stores for the circulating excess nutrients. This increased storage is accompanied by secretion of multiple adipokines (adipocyte-derived hormones) that can affect whole-body metabolism as well as recruit immune cells to adipose tissue $[3,4]$. Immune infiltration into adipose tissue has been linked to a plethora of complications including insulin resistance, cardiovascular diseases, metabolic syndrome and type-2 diabetes [5].

Acylation stimulating protein (ASP, aka C3adesArg) is one such adipokine that correlates with various metabolic and hormonal disturbances. Plasma levels are increased with insulin resistance, cardiovascular disease, metabolic syndrome, type 2 diabetes and polycystic ovary disease, even in the absence of obesity. Dietary lipoproteins (in the form of chylomicrons), as well as insulin, increase C3 and ASP production in vivo, and plasma ASP correlates with fat clearance in vivo [6,7]. On the other hand, plasma ASP concentrations diminish following diet, exercise or bariatric surgery $[8,9]$.

ASP is derived from complement component C3 which, when activated, is cleaved to produce the anaphylatoxins $\mathrm{C} 3 \mathrm{a}$ and $\mathrm{C} 3 \mathrm{~b}$ (which can then proceed to initiate the alternative complement immune pathway). In circulation, C3a rapidly undergoes $\mathrm{C}$-terminal cleavage via carboxypeptidase $\mathrm{B}$ to produce C3adesArg (ASP) [8]. The amount of C3adesArg/ASP generated will depend on the local C3 concentration as well as the rate of enzymatic conversion of C3 to ASP. Adipose tissue C3 mRNA increases in obesity and correlates with body mass index (BMI), however, the proportion of ASP relative to C3 in plasma is low 
$(<0.5 \%)$, suggesting that there is stringent regulation of conversion from C3 to ASP [10].

ASP has been shown to act through a 7-transmembrane receptor (C5L2) to stimulate fatty acid uptake and esterification as well as glucose transport into adipocytes while decreasing lipolysis via inhibition of hormone-sensitive lipase. These effects are independent and additive to those of insulin [8]. C5L2 receptor is present in adipocytes, but also in muscle, brain, spleen, liver and immune cells [11]. In addition to lipogenic effects, recent studies have shown that ASP can increase adipocyte production of other adipokines as well as inflammatory factors, including macrophage chemoattractant protein-1 (MCP-1) and KC (murine ortholog of IL-8). These pro-inflammatory factors are partly responsible for the recruitment of macrophages into adipose tissue, thus initiating interaction between adipose and immune cells. Based on these results, we set out to identify other inflammatory and metabolic hormones that would affect ASP production using a 3T3-L1 cell model.

\section{Materials and Methods \\ $\nabla$}

\section{Materials}

Reagents were obtained as follows: leptin, TNF $\alpha$, IL-10, adiponectin, KC and RANTES were purchased from ProSpec-Tany TechnoGene Ltd (Rehovot, Israel), LPS from Sigma-Aldrich (Oakville, ON, Canada). Apelin and Visfatin were obtained from Phoenix Pharmaceuticals Inc (Burlingame, CA, USA). Omentin was obtained from Enzo Life Sciences Inc in Farmingdale, NY, USA and C5a was acquired at VWR in Mississauga, ON, Canada. All tissue culture reagents, including Dulbeccos's modified Eagle's medium/F-12 (DMEM/F12), phosphate buffered saline (PBS), fetal bovine serum (FBS), trypsin and tissue culture supplies were from Gibco (Burlington, ON, Canada). Triglyceride (TG) mass was measured using an enzymatic colorimetric assay (Roche Diagnostic, Indianapolis, IN, USA).

\section{Cell culture and 3T3-L1 differentiation}

3T3-L1 preadipocytes and J774 macrophages were purchased from the American Type Culture Collection (Manassas, VA, USA). Cells were cultured and differentiated as described previously [6]. Briefly; Preadipocytes (3T3-L1) were plated in 24-well culture plates $\left(\approx 3 \times 10^{4}\right.$ cells $/$ welL $)$ and differentiated with medium containing $10 \mu \mathrm{g} / \mathrm{ml}$ insulin, $1 \mu \mathrm{M}$ dexamethasone and $500 \mu \mathrm{M}$ isobutylmethylxanthine (IBMX). Differentiated cells, at least $90 \%$ of which showed an adipocyte phenotype by accumulation of lipid droplets (as determined by microscopic evaluation), were used on days 8-12 after initiation of differentiation. J774 macrophages were plated in 12 -well culture plates $\left(\approx 1 \times 10^{5}\right.$ cells $/$ well; trypan blue exclusion) and used within 2-3 days.

\section{Direct and indirect co-culture of adipocytes and macrophages}

Direct coculture was performed by adding J774 macrophages $\left(\approx 1 \times 10^{5}\right.$ viable cells/well; trypan blue exclusion) into 12 -well culture plates containing differentiated 3T3-L1 adipocytes $\left(\approx 1 \times 10^{5}\right.$ cells/well). The same numbers of J774 and 3T3-L1 cells were also plated separately in different wells for comparison. Adipocytes and macrophages were in contact in fresh serumfree medium for $2 \mathrm{~h}$ before addition of any treatments.

\section{Cell treatments}

Adipocytes, macrophages and co-cultured cells were incubated for $2 \mathrm{~h}$ in serum-free medium (DMEM/F12) prior to stimulation at various physiological concentrations with the treatments as indicated. All of the treatments were conducted in serum free medium. After $24 \mathrm{~h}$ of treatment, the culture supernatants were collected and stored at $-80^{\circ} \mathrm{C}$ until analysis.

\section{Medium ASP and C3 determination}

Mouse ASP (C3a) ELISA reagents were from BD Pharmingen (Franklin Lakes, NJ, USA). ASP was measured using a sandwich ELISA as described previously [6]. Note, the antibody used in this ELISA is specific for a neoepitope exposed in mouse C3a and its desArginated form (ASP), but does not cross-react with the native protein C3. A mouse complement C3 ELISA kit (Kamiya Biomedical, Seattle, WA, USA) was used to measure total C3, including native and breakdown products, and was used according to the manufacturer's instructions with calculation by linear regression against a standard curve. TG mass was measured using an enzymatic colorimetric assay (Roche Diagnostics, Indianapolis, IN, USA).

\section{Statistical analyses}

Results are presented as mean \pm SEM. Each experiment was performed at least 3 times, with individual triplicate analyses in each experiment $(n=9)$. ASP and C3 production were quantified in each experiment, normalized to cell TG mass (nmol/well), and reported relative to the untreated control $(\mathrm{CTL}=100 \%)$, which was cells incubated in serum-free medium alone. The proportion of C3 converted to ASP is calculated as \% ASP/C3 (ng/ng). Comparison between 2 groups was determined by Student's $t$-test and comparisons amongst several groups were performed by 1-way ANOVA followed by Newman-Keuls or Dunnett's multiple comparison post-test using Prism software (Graphpad Software). Statistical significance was set at $P<0.05$, where NS indicates not significant relative to the indicated control.

\section{Results}

\section{$\nabla$}

\section{Effects of adiponectin and leptin on ASP and C3} secretion in 3T3-L1 adipocytes

ASP production and C3 secretion stimulated by leptin and adiponectin (globular domain gAcrp30 and full-length high molecular weight (HMW) adiponectin) are shown in $\bullet$ Fig. 1, 2 . The lowest concentration of leptin $(10 \mathrm{ng} / \mathrm{ml})$ had no effect on ASP production but there was a significant increase in ASP production with higher concentrations ( $\odot$ Fig. 1a; $100 \mathrm{ng} / \mathrm{mL}, 151 \%$, $P<0.01$ and $1000 \mathrm{ng} / \mathrm{mL}, 132 \%, P<0.05)$. C3 secretion increased slightly at $10 \mathrm{ng} / \mathrm{ml}$ and $1000 \mathrm{ng} / \mathrm{ml}$ of leptin treatment but was only significantly increased at $100 \mathrm{ng} / \mathrm{ml}$ ( $\odot$ Fig. 2a; $165 \%$, $P<0.05$ ). The ratio of ASP to C3, which reflects the conversion rate of C3 to ASP was constant at all leptin concentrations used (data not shown).

Both HMW adiponectin (HMW previously defined above) adiponectin and globular (gAcrp) adiponectin were used to treat differentiated 3T3-L1 adipocytes. The highest concentration of HMW adiponectin $(5 \mathrm{ug} / \mathrm{ml})$ increased ASP production and C3 secretion in 3T3-L1 adipocytes ( $\bullet$ Fig. $\mathbf{1 b}, \mathbf{2 b} ; \mathbf{1 5 3} \%, 152 \%$, $P<0.05$, respectively). There was no effect on either ASP production or $\mathrm{C} 3$ secretion at both concentrations of gAcrp $(\bullet$ Fig. 1b, 2b; 


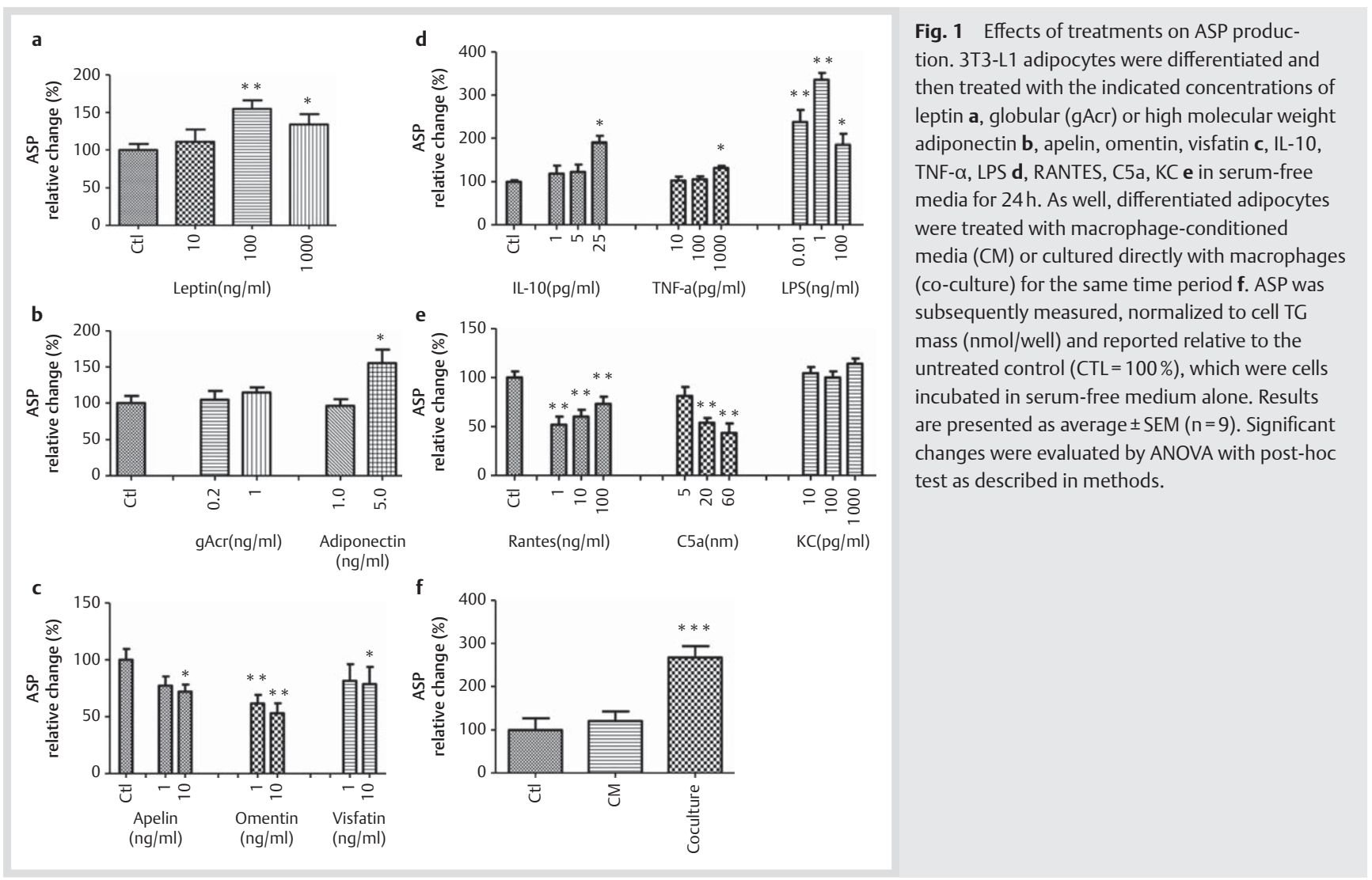

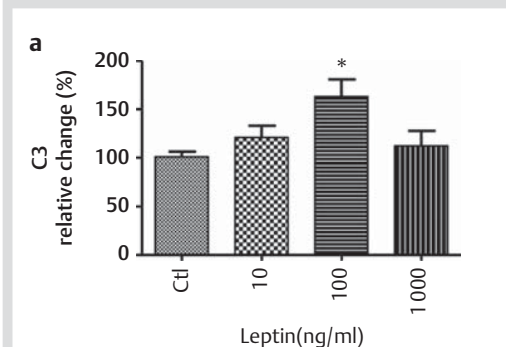

b
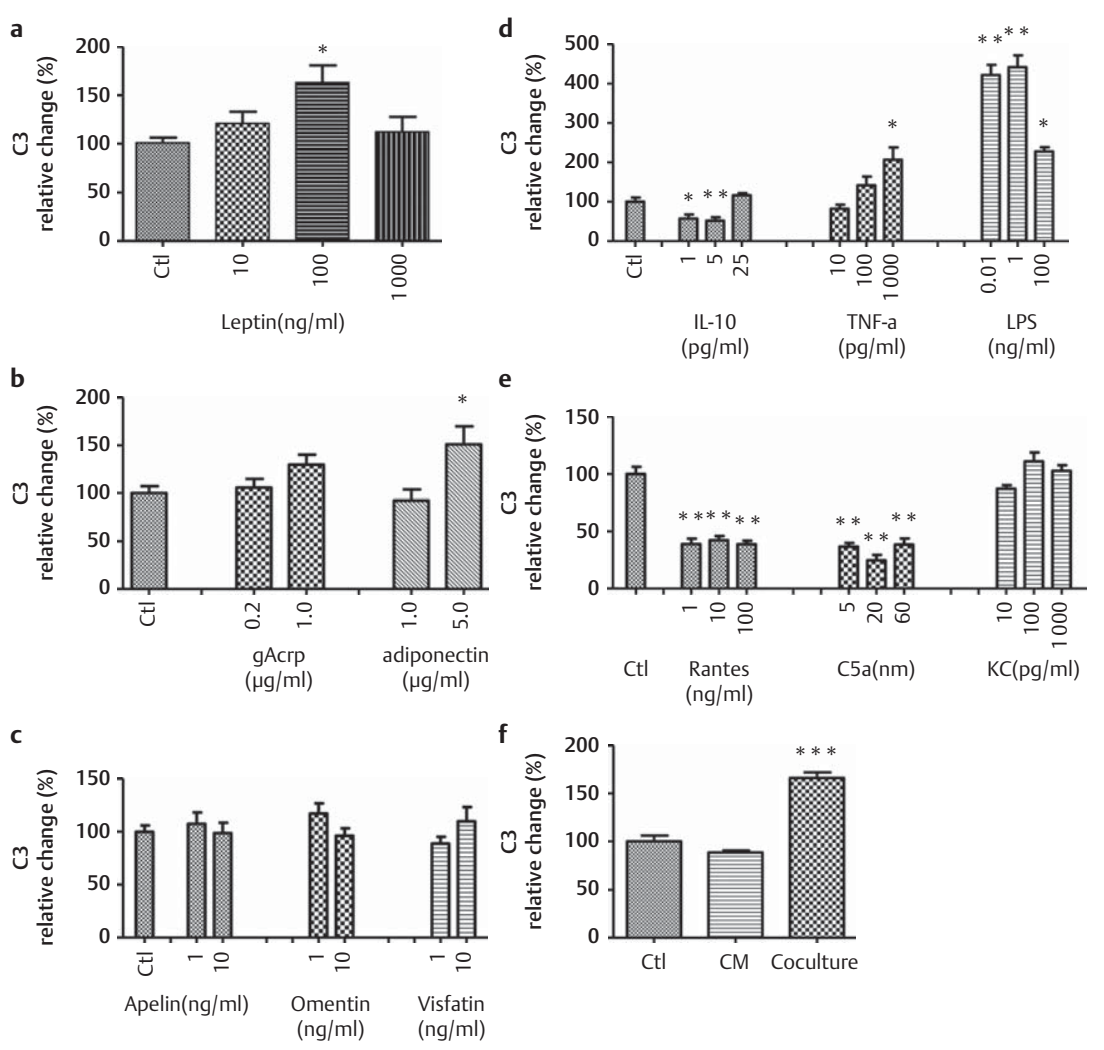

Fig. 2 Effects of treatments on C3 secretion. 3T3-L1 adipocytes were differentiated and then treated with the indicated concentrations of leptin a, globular ( $\mathrm{gAcr}$ ) or high molecular weight adiponectin $\mathbf{b}$, apelin, omentin, visfatin $\mathbf{c}$, IL-10, TNF- $\alpha$, LPS d, RANTES, C5a, KC e in serum-free media for $24 \mathrm{~h}$. As well, differentiated adipocytes were treated with macrophage-conditioned media or cultured directly with macrophages (co-culture) for the same time period $\mathbf{f}$. $\mathrm{C} 3$ was subsequently measured, normalized to cell TG mass (nmol/well) and reported relative to the untreated control (CTL $=100 \%$ ), which were cells incubated in serum-free medium alone. Results are presented as average $\pm \operatorname{SEM}(n=9)$. Significant changes were evaluated by ANOVA with post-hoc test as described in methods. 


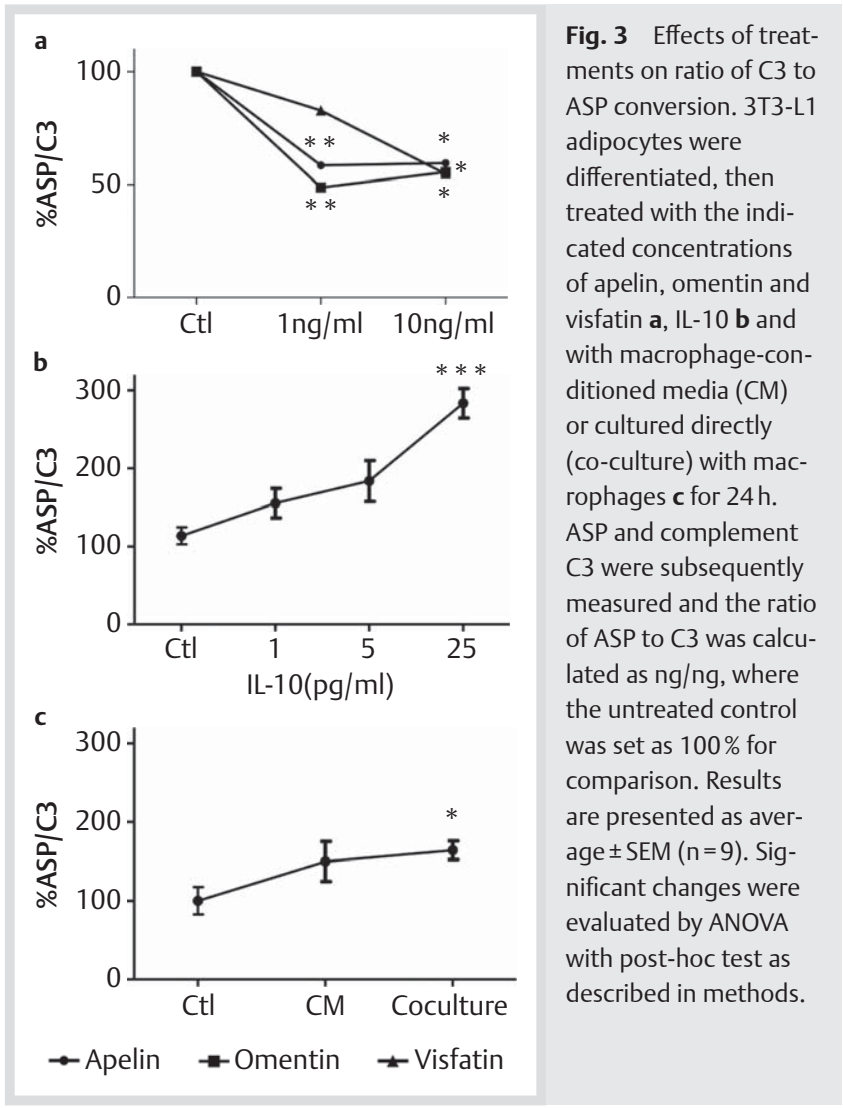

$0.2 \mathrm{ug} / \mathrm{mL}$ and $1 \mathrm{ug} / \mathrm{mL}$ ). The conversion of C3 to ASP (\%ASP/C3) did not change (data not shown). These results suggest that fulllength adiponectin and leptin could stimulate ASP production in adipocytes by increasing precursor $\mathrm{C} 3$ production rather than conversion.

Effects of novel adipokines apelin, omentin and visfatin on ASP and C3 secretion in 3T3-L1 adipocytes

ASP production and C3 secretion subsequent to treatment with apelin, omentin and visfatin were also evaluated. Both concentrations $(1 \mathrm{ng} / \mathrm{mL}, 10 \mathrm{ng} / \mathrm{mL})$ of omentin decreased ASP production but only the highest concentration of apelin and visfatin $(10 \mathrm{ng} / \mathrm{ml})$ significantly decreased ASP production in 3T3-L1 adipocytes ( $\odot$ Fig. 1c; $P<0.05, P<0.01$, respectively). C3 secretion did not significantly change at any treatment concentration with the adipokines tested ( $P$ NS; $\odot$ Fig. $2 \mathrm{c}$ ). The ratio of ASP to C3 was also decreased at both concentrations of apelin and omentin as well as at the highest concentration of visfatin $\left(10^{`} \mathrm{ng} / \mathrm{ml}\right)$ $(\odot$ Fig. 3a; $P<0.05$ ). The results suggest that apelin, omentin and visfatin could inhibit ASP production in adipocytes by decreasing the rate of $\mathrm{C} 3$ conversion.

\section{Effects of inflammatory factors IL-10, TNF- $\alpha$ and lipopolysaccharide (LPS) on ASP production and C3 secretion in 3T3-L1 adipocytes}

ASP and C3 secretion stimulated by IL-10, TNF- $\alpha$ and LPS are also shown here. Only the highest concentrations of IL-10 $(25 \mathrm{pg} / \mathrm{ml})$ and TNF- $\alpha(1000 \mathrm{pg} / \mathrm{ml})$ increased ASP production in 3T3-L1 adipocytes ( $\odot$ Fig. 1d; $190 \%, 134 \%, P<0.05$, respectively). With LPS treatment, all concentrations strongly stimulated ASP production ( $\odot$ Fig. 1d; $0.01 \mathrm{ng} / \mathrm{mL}, 220 \%, P<0.001 ; 1 \mathrm{ng} / \mathrm{mL}, 318 \%$, $P<0.001 ; 100 \mathrm{ng} / \mathrm{mL}, 188 \%, P<0.01)$.
Interestingly, IL-10 treatment decreased C3 production at lower concentrations ( $\odot$ Fig. 2 d; 1 pg/mL, $P<0.05 ; 5 \mathrm{pg} / \mathrm{ml}, P<0.01$ ) but there was no change in $\mathrm{C} 3$ production at the highest concentration $(25 \mathrm{pg} / \mathrm{ml})$. For TNF- $\alpha$ and LPS treatments, changes in C3 secretion followed the same direction as the ASP changes: the highest concentration of TNF- $\alpha$ increased C3 secretion ( $\bullet$ Fig. 2d; $210 \%, P<0.05)$ while all concentrations of LPS strongly stimulated C3 secretion ( $\odot$ Fig. 2 d; $415 \%, P<0.001 ; 423 \%, P<0.001$; $215 \%, P<0.01$ ).

The ratio of ASP to C3 is significantly increased for the highest IL-10 concentration, with a concentration dependent effect ( $\odot$ Fig. 3b; $P<0.001$ ), suggesting that relative ASP production changed less at lower IL-10 levels, but is increased with a supraphysiological concentration. With LPS treatment, both ASP and C3 increased, and the relative ASP/C3 ratio was unchanged (data not shown). Although overall, TNF- $\alpha$ stimulated both $\mathrm{C} 3$ and $\mathrm{ASP}$, the ratio of $\mathrm{ASP} / \mathrm{C} 3$ varied, with an increase in $\mathrm{ASP} / \mathrm{C} 3$ at the lowest concentration of TNF- $\alpha(10 \mathrm{pg} / \mathrm{ml})$, and a relative decrease at the highest concentration (data not shown).

\section{Effects of inflammatory factors RANTES, C5a and KC on} ASP and C3 secretion in 3T3-L1 adipocytes

In contrast to the stimulatory effects of IL-10, TNF $\alpha$ and LPS, there was a decrease in ASP production when RANTES and C5a were used. All concentrations of RANTES decreased ASP production ( $\odot$ Fig. 1e; all $P<0.01$,) and changes in C3 secretion followed the same direction ( $\odot$ Fig. 2 e all $P<0.01$ ). Higher concentrations of C5a (20 nM and $60 \mathrm{nM})$ significantly decreased ASP production ( $\odot$ Fig. 1 e; both $P<0.001$ ) and all C5a concentrations strongly decreased C3 secretion ( $\bullet$ Fig. 2e; all $P<0.001$ ). There was no effect on ASP, and C3 secretion at any concentration of KC ( $\odot$ Fig. 1e, 2e; $P$ NS,). As the changes in ASP and C3 paralleled each other, the ratio of ASP/C3 was unchanged for RANTES, C5a or KC treatments (data not shown). These results suggest that RANTES and C5a may both inhibit ASP production in adipocytes by decreasing precursor $\mathrm{C} 3$ secretion.

\section{Effects of direct co-culture with macrophages and macrophage-conditioned media on ASP and C3 secretion in 3T3-L1 adipocytes}

We evaluated the effects of macrophages directly on ASP and C3 in 3T3-L1 adipocytes. Macrophage conditioned media (CM) was collected after overnight treatment of J774 in serum-free media. No ASP or C3 was detectable in the conditioned-media. Further, conditioned-media had no effect on secretion of $\mathrm{C} 3$, production of ASP or the ASP/C3 ratio in 3T3-L1 adipocytes ( $\bullet$ Fig. 1f, 2f, 3c; $P N S$ ). However, direct co-culture of adipocytes with J774 cells enhanced secretion of $\mathrm{C} 3$ and production of ASP in adipocytes. Moreover, the relative increase in ASP ( $\odot$ Fig. 1f; $272 \%, P<0.001$ ) was much higher than that of C3 secretion ( 0 Fig. 2 f; $167 \%$, $P<0.001$ ), which reflects a higher conversion of C3 to ASP. These results suggest that increased ASP levels are a result of increased precursor levels as well as increased rates of conversion ( $\bullet$ Fig. 3c; $162 \%, P<0.05)$.

\section{Discussion}

\section{$\nabla$}

Here, the effects of various physiological concentrations of cytokines and hormones involved in inflammatory processes or metabolic disturbances on the production of ASP and its precursor (complement C3) were tested in differentiated adipocytes. 
Furthermore, we measured ASP production in a cell model mimicking an obese environment: co-culture of adipocytes with macrophages, or treatment with macrophage conditioned media. These novel results indicate that various metabolic hormones and inflammatory factors have varying effects on ASP and C3 production in 3T3-L1 adipocytes. The overall pattern was that inflammatory factors stimulated ASP secretion in 3T3-L1 adipocytes while most of the insulin sensitizing hormones decreased its secretion (with the exception of adiponectin), and the implications of this are discussed below both, first for the individual factors in relationship to physiological data, as well as overall.

However, there are some limitations in this study that should be acknowledged. First, 3T3-L1 adipocytes and J774 macrophages are murine cell lines, and do not represent the heterogeneity which is found in adipocytes derived from humans. The co-culture and conditioned media approaches were used to mimic the situation in adipose tissue from obese subjects, where macrophage infiltration is present. Nonetheless, this still represents an imperfect scenario, and does not capture the adipose tissue inflammation observed in obese subjects in relation to heterogeneity in adipocyte size, macrophage phenotype and even gender and age. Further, we only detected ASP and C3 secretion for $24 \mathrm{~h}$ of treatment and long-term effects of these treatments have yet to be elucidated.

\section{The effects of the metabolic hormones apelin, visfatin and omentin on ASP and C3 production}

Apelin is mainly produced and secreted by adipocytes, and stimulates glucose uptake through the PI3K/Akt pathway, improving insulin resistance in 3T3-L1 adipocytes [12]. Visfatin, mainly produced by lymphocytes, decreases insulin resistance: administration in mice decreases blood glucose levels, whereas mice lacking one allele have increased plasma glucose [13]. Omentin, an adipokine preferentially produced by visceral adipose tissue, has insulin-sensitizing effects, and circulating levels are decreased in insulin-resistant states [14]. Altogether, these adipokines exert beneficial effects in metabolic syndrome and all have similar effects of diminishing ASP production via lowering C3 conversion rates (with no effect on C3 secretion).

\section{The effects of anti-inflammatory markers on ASP and C3 production}

Many studies demonstrate that adiponectin has direct anti-diabetic, anti-atherogenic and anti-inflammatory functions $[15,16]$. Adiponectin can exist as a full-length or a smaller, globular fragment. While most plasma adiponectin appears to be in the fulllength isoform, forming multimers as 3 different oligomeric complexes, high molecular weight (HMW), middle molecular weight (MMW) and low molecular weight (LMW), a small amount of globular adiponectin (gAcrp) can be detected in human plasma. Although adiponectin improves insulin resistance, similar to the adipokines mentioned above, by contrast it did not have the same effects on ASP and C3 production. Interestingly, in obese subjects, plasma adiponectin decreases, while other insulin sensitizing hormones such as apelin are increased in obese subjects. An alternate link between adiponectin and ASP may be found in the study by Peake et al., which showed that adiponectin was bound by the globular domain of the A chain of collagenase-digested complement $\mathrm{C} 1 \mathrm{q}$, and $\mathrm{C} 1 \mathrm{q}$ binding induced deposition of $\mathrm{C} 4$ and $\mathrm{C} 3$ through activation of the classical complement pathway [17]. As a member of the pattern-rec- ognition family of defense collagens, adiponectin binds targeted molecules and activates complement. Thus, it could directly stimulate $\mathrm{C} 3$ production and cleavage to increase ASP production (as demonstrated here).

Interleukin (IL)-10 is a major anti-inflammatory cytokine that plays a crucial role in the regulation of the immune system. High levels of circulating IL-10 are associated with obesity and metabolic syndrome $[18,19]$. Moreover IL-10 levels decrease following weight loss [20]. We found that IL-10 could directly inhibit C3 secretion in adipocytes. However, ASP levels do not decrease (relative to control), probably due to an increase in C3 conversion to ASP. This is also consistent with in vivo studies, which have shown that both ASP and IL-10 are increased in obesity and infectious diseases, although the underlying mechanisms are still unclear.

\section{The effects of pro-inflammatory markers on ASP and C3 production}

Leptin is one of the more prominently studied adipokines, inhibiting food consumption and stimulating energy expenditure [21]. Moreover leptin also has a pro-inflammatory effect [22]. In the present study, a high concentration of leptin increased ASP and $\mathrm{C} 3$ secretion, which is consistent with previous in vivo studies where subjects with obesity, diabetes and cardiovascular disease have both increased leptin and ASP $[8,23]$.

Tumour necrosis factor-alpha (TNF- $\alpha$ ) is a proinflammatory cytokine secreted by various cells including monocytes, macrophages and adipocytes [24,25] with roles in acute phase response, lipid metabolism and adipocyte function [26-28]. Plasma TNF- $\alpha$ levels and expression of TNF- $\alpha$ in adipose tissue are increased in obesity and correlate with insulin resistance [29]. In this study, we found that the highest concentration of TNF- $\alpha(1000 \mathrm{pg} / \mathrm{ml})$ increased C3 and ASP secretion, although the ASP increase was not proportional to the $\mathrm{C} 3$ increase.

Lipopolysaccharide (LPS) is a strong inducer of inflammation and was shown to trigger secretion of immunomodulatory molecules such as TNF- $\alpha$, IL-6 and MCP-1 in differentiated 3T3-L1 adipocytes via toll-like receptor activation [30]. LPS administration, a widely used experimental model of infection and inflammation, also induces hyperglycemia and insulin resistance in mice $[31,32]$. Physiologically, circulating LPS increases in Type 2 diabetes, and is preferentially associated with postprandial chylomicrons [30], which, in turn, increase C3 secretion and C3 conversion to ASP [33]. The present study shows that ASP production and C3 secretion were increased 2-4 fold, even at very low LPS concentrations $(0.01 \mathrm{ng} / \mathrm{ml})$.

\section{The effects of chemokines on ASP and C3 production}

Chemokines produced by fat, including C5a, IL-8, MCP-1, and RANTES are often regulated by adipokines [34]. Links between RANTES and C3 have been described previously: serum RANTES is increased in systemic lupus erythematosus and correlates with C3 levels [35]; C3 and C5 deficient mice have decreased RANTES and MCP1, and treatment with C3a or C5a caused upregulation of IL-8, IL-1ß, and RANTES mRNA in a time- and dosedependent manner in human umbilical cord endothelial cells [36]. These results suggest that $\mathrm{C} 3$ could contribute to the positive regulation of RANTES production. C3 activation also leads to complement cascade responses to produce the chemoattractants C3a and C5a. We speculate that there may exist a feedback regulation effect of RANTES and C5a on adipocyte C3 production. Lower levels of $\mathrm{C} 3$ would then be reflected in lower levels of 
circulating ASP, as shown here. An additional point that should be highlighted here is that $\mathrm{C} 5 \mathrm{~L} 2$, the second receptor of $\mathrm{C} 5 \mathrm{a}$, is also a functional receptor for ASP [11]. Recent reports suggest that C5L2 plays an active, positive role in inflammatory responses [37]. Thus increases in C5a could translate into higher binding with C5L2, causing internalization, which might then lead to less C5L2 being available for ASP binding, also interfering with ASP function in this manner.

\section{Direct co-culture of adipocytes with macrophages enhance ASP and C3 secretion}

The present results demonstrate that numerous hormones, proand anti-inflammatory factors and chemokines have varying effects on ASP production via effects on precursor C3 or C3 conversion to ASP. In obesity, immune cell infiltration into adipose tissue results in increased secretion of many of these same factors $[38,39]$. While in the present study, macrophage-conditioned media alone had little effect on C3 or ASP, the co-culture of adipocytes with macrophages markedly altered ASP production. This suggests that there is a strong local crosstalk between these 2 types of cells. The process of macrophage infiltration into adipose tissue could be mediated by the secretion of chemokines (such as TNF- $\alpha$, MCP- 1 and KC) by activated adipocytes, and at the same time the infiltration of macrophages into adipose tissue would then be an important source of TNF- $\alpha$, IL- 6 and other inflammatory factors [40]. Thus, direct co-culture with macrophages could lead to a vicious cycle of pro-inflammatory activation, which leads to synergistic effects on increased ASP and $\mathrm{C} 3$ production in adipocytes.

In light of these differential effects of adipokines, pro- and antiinflammatory factors and chemokines on ASP and C3, the relationship of ASP to various metabolic and hormonal dysfunctions is pertinent. Plasma ASP levels are increased in obesity, insulin resistance and related diseases such as cardiovascular disease, type 2 diabetes and polycystic ovary syndrome [33,41-43]. Further, increased fasting plasma ASP is associated with a delay in postprandial triglyceride clearance in humans [8]. In vivo, this is also accompanied by reduced specific binding and response to ASP in cells from subjects with high ASP levels [42]. These increased ASP levels, accompanied with decreased receptor expression and ASP response, suggest that there exists an ASP resistance state. Further, ASP could be a novel pro-inflammatory factor that stimulates secretion of certain cytokines and activates the NF-KB signalling pathway [44]. Thus, ASP is a biomarker for abnormal lipid metabolism and accumulation of fat tissue, where high ASP levels are associated with increased risk factors for metabolic abnormalities.

In summary, various metabolic hormones and inflammatory factors can affect ASP production through increased precursor C3 production and/or by changing the rate of $\mathrm{C} 3$ conversion to ASP. As an adipokine, ASP could constitute an additional link between adipose tissue and immune systems. Moreover, the crosstalk between adipocytes and macrophages enhances ASP and $\mathrm{C} 3$ production, further promoting inflammation in adipose tissue, especially in states of obesity and its various associated complications.

\section{Grants \& Fellowships}

$\nabla$

K. Cianflone holds a Canada Research Chair in Adipose Tissue. This study was supported by a grant from CIHR (to KC) and a grant from National Natural Science Foundation of China (NSFC) (\#81270949 to HL and \#81170627 to XPL). Danny Gauvreau was supported by a fellowship from Centre de Recherche de l'Institut Universitaire de Cardiologie et Pneumologie de Quebec.

\section{Acknowledgements}

$\nabla$

The authors would like to thank Mélanie Cianflone for administrative assistance in preparation of this manuscript.

Conflict of interest disclosure: The authors have no conflict of interest to disclose.

\section{References}

1 Weisberg SP, McCann D, Desai M et al. Obesity is associated with macrophage accumulation in adipose tissue. J Clin Invest 2003; 112: 1796-1808

2 Hotamisligil GS, Arner P, Caro JF et al. Increased adipose tissue expression of tumor necrosis factor-alpha in human obesity and insulin resistance. J Clin Invest 1995; 95: 2409-2415

3 Sartipy P, Loskutoff DJ. Expression profiling identifies genes that continue to respond to insulin in adipocytes made insulin-resistant by treatment with tumor necrosis factor-alpha. J Biol Chem 2003; 278: 52298-52306

4 Kim CS, Park HS, Kawada T et al. Circulating levels of MCP-1 and IL-8 are elevated in human obese subjects and associated with obesityrelated parameters. Int J Obes (Lond) 2006; 30: 1347-1355

5 Zeyda M, Stulnig TM. Adipose tissue macrophages. Immunol Lett 2007; 112: 61-67

6 Gao Y, Gauvreau D, Cianflone K. Hormone and pharmaceutical regulation of ASP production in 3T3-L1 adipocytes. J Cell Biochem 2010; 109: 896-905

7 Murray I, Sniderman AD, Cianflone K. Enhanced triglyceride clearance with intraperitoneal human acylation stimulating protein in C57BL/6 mice. Am J Physiol 1999; 277: E474-E480

8 Cianflone K, Xia Z, Chen LY. Critical review of acylation-stimulating protein physiology in humans and rodents. Biochim Biophys Acta 2003; 1609: 127-143

9 Schrauwen P, Hesselink MK, Jain M et al. Acylation-stimulating protein: effect of acute exercise and endurance training. Int J Obes (Lond) 2005; 29: 632-638

10 Cianflone K, Maslowska M. Differentiation-induced production of ASP in human adipocytes. Eur J Clin Invest 1995; 25: 817-825

11 Kalant D, MacLaren R, Cui $W$ et al. C5L2 is a functional receptor for acylation-stimulating protein. J Biol Chem 2005; 280: 23936-23944

12 Zhu S, Sun F, Li W et al. Apelin stimulates glucose uptake through the PI3K/Akt pathway and improves insulin resistance in 3T3-L1 adipocytes. Mol Cell Biochem 2011; 353: 305-313

13 Sommer G, Garten A, Petzold S et al. Visfatin/PBEF/Nampt: structure, regulation and potential function of a novel adipokine. Clin Sci (Lond) 2008; 115: 13-23

14 Yang $\mathrm{RZ}$, Lee MJ, Hu $\mathrm{H}$ et al. Identification of omentin as a novel depot-specific adipokine in human adipose tissue: possible role in modulating insulin action. Am J Physiol Endocrinol Metab 2006; 290: E1253-E1261

15 Whitehead JP, Richards AA, Hickman IJ et al. Adiponectin - a key adipokine in the metabolic syndrome. Diabetes Obes Metab 2006; 8: 264-280

16 Brochu-Gaudreau K, Rehfeldt C, Blouin $R$ et al. Adiponectin action from head to toe. Endocrine 2010; 37: 11-32

17 Peake PW, Shen Y, Walther A et al. Adiponectin binds C1q and activates the classical pathway of complement. Biochem Biophys Res Commun 2008; 367: 560-565

18 Bluher M, Fasshauer M, Tonjes A et al. Association of interleukin-6, C-reactive protein, interleukin-10 and adiponectin plasma concentrations with measures of obesity, insulin sensitivity and glucose metabolism. Exp Clin Endocrinol Diabetes 2005; 113: 534-537

19 Calcaterra V, De Amici M, Klersy C et al. Adiponectin, IL-10 and metabolic syndrome in obese children and adolescents. Acta Biomed 2009; 80: $117-123$

20 Dalmas E, Rouault C, Abdennour $M$ et al. Variations in circulating inflammatory factors are related to changes in calorie and carbohydrate intakes early in the course of surgery-induced weight reduction. Am J Clin Nutr 2011; 94: 450-458 
21 Belgardt BF, Bruning JC. CNS leptin and insulin action in the control of energy homeostasis. Ann N Y Acad Sci 2010; 1212: 97-113

22 Agrawal S, Gollapudi S, Su H et al. Leptin activates human B cells to secrete TNF-a, IL-6, and IL-10 via JAK2/STAT3 and p38MAPK/ERK1/2 signaling pathway. J Clin Immunol 2011; 31: 472-478

23 Tulipano $G$, Vergoni $A V$, Soldi $D$ et al. Characterization of the resistance to the anorectic and endocrine effects of leptin in obesity-prone and obesity-resistant rats fed a high-fat diet. J Endocrinol 2004; 183 : 289-298

24 Waage A. Tumour necrosis factor and septic shock. Lancet 1998; 351: 603

25 Sewter CP, Digby JE, Blows $F$ et al. Regulation of tumour necrosis factor-alpha release from human adipose tissue in vitro. J Endocrinol 1999; 163: 33-38

26 Medina EA, Stanhope KL, Mizuno TM et al. Effects of tumor necrosis factor alpha on leptin secretion and gene expression: relationship to changes of glucose metabolism in isolated rat adipocytes. Int J Obes Relat Metab Disord 1999; 23: 896-903

27 Rosenstock M, Greenberg AS, Rudich A. Distinct long-term regulation of glycerol and non-esterified fatty acid release by insulin and TNF-alpha in 3T3-L1 adipocytes. Diabetologia 2001; 44: 55-62

28 Hirokawa J, Sakaue S, Furuya $Y$ et al. Tumor necrosis factor-alpha regulates the gene expression of macrophage migration inhibitory factor through tyrosine kinase-dependent pathway in 3T3-L1 adipocytes. J Biochem 1998; 123: 733-739

29 Winkler G, Lakatos P, Salamon $F$ et al. Elevated serum TNF-alpha level as a link between endothelial dysfunction and insulin resistance in normotensive obese patients. Diabet Med 1999; 16: 207-211

30 Creely SJ, McTernan PG, Kusminski CM et al. Lipopolysaccharide activates an innate immune system response in human adipose tissue in obesity and type 2 diabetes. Am J Physiol Endocrinol Metab 2007; 292: E740-E747

31 Vitseva OI, Tanriverdi K, Tchkonia TT et al. Inducible Toll-like receptor and NF-kappaB regulatory pathway expression in human adipose tissue. Obesity (Silver Spring) 2008; 16: 932-937

32 Kopp A, Buechler C, Neumeier $M$ et al. Innate immunity and adipocyte function: ligand-specific activation of multiple Toll-like receptors modulates cytokine, adipokine, and chemokine secretion in adipocytes. Obesity (Silver Spring) 2009; 17: 648-656
33 Yang Y, Lu HL, Zhang J et al. Relationships among acylation stimulating protein, adiponectin and complement $\mathrm{C} 3$ in lean vs obese type 2 diabetes. Int J Obes (Lond) 2006; 30: 439-446

34 Gerhardt CC, Romero IA, Cancello $R$ et al. Chemokines control fat accumulation and leptin secretion by cultured human adipocytes. Mol Cell Endocrinol 2001; 175: 81-92

$35 \mathrm{Lu}$ MM, Wang J, Pan HF et al. Increased serum RANTES in patients with systemic lupus erythematosus. Rheumatol Int 2012; 32: 1231-1233

36 Sewell DL, Nacewicz B, Liu F et al. Complement C3 and C5 play critical roles in traumatic brain cryoinjury: blocking effects on neutrophil extravasation by C5a receptor antagonist. J Neuroimmunol 2004; 155 : 55-63

37 Ward PA. Functions of C5a receptors. J Mol Med (Berl) 2009; 87: 375-378

38 Suganami T, Ogawa Y. Adipose tissue macrophages: their role in adipose tissue remodeling. J Leukoc Biol 2010; 88: 33-39

$39 \mathrm{Xu} \mathrm{H}$, Barnes GT, Yang $Q$ et al. Chronic inflammation in fat plays a crucial role in the development of obesity-related insulin resistance. I Clin Invest 2003; 112: 1821-1830

40 Harford KA, Reynolds CM, McGillicuddy FC et al. Fats, inflammation and insulin resistance: insights to the role of macrophage and T-cell accumulation in adipose tissue. Proc Nutr Soc 2011; 70: 408-417

41 Maslowska $\mathrm{M}, \mathrm{Vu} \mathrm{H}$, Phelis $\mathrm{S}$ et al. Plasma acylation stimulating protein, adipsin and lipids in non-obese and obese populations. Eur J Clin Invest 1999; 29: 679-686

42 Cianflone K, Zhang XJ, Genest J Jr et al. Plasma acylation-stimulating protein in coronary artery disease. Arterioscler Thromb Vasc Biol 1997; 17: 1239-1244

$43 \mathrm{Wu} Y$, Zhang J, Wen $Y$ et al. Increased acylation-stimulating protein, C-reactive protein, and lipid levels in young women with polycystic ovary syndrome. Fertil Steril 2009; 91: 213-219

44 MacLaren RE, Cui W, Lu H et al. Association of adipocyte genes with ASP expression: a microarray analysis of subcutaneous and omental adipose tissue in morbidly obese subjects. BMC Med Genomics 2010; $3: 3$ 